\title{
医药健康领域的国家人工智能战略发展规划比较研究
}

\author{
李芗芗 $^{1}$, 张建楠 ${ }^{1}$, 顾宴菊 ${ }^{1}$, 朱烨琳 ${ }^{1}$, 何前锋 ${ }^{2}$, 李兰娟 ${ }^{1,2}$ \\ (1. 浙江数字医疗卫生技术研究院, 杭州 $311100 ； 2$. 浙江大学医学院附属第一医院, 杭州 310003）
}

摘要: 本文梳理比较了 2015 年以来在医药健康领域有重点布局人工智能技术发展的美国、日本、英国、印度及我国的战略 规划情况。分析显示, 基础建设投入和基于国情的重点领域布局是战略规划的核心内容, 我国战略布局多方面仅次于美国, 但相对缺乏整体规划与发展路径, 建设重点有待明确, 健康医疗数据基础未与人工智能应用发展形成有效衔接、复合人才结 构失衡的问题未充分重视、人工智能应用的监管及制度规范亟待加强。为推动我国人工智能在医药健康领域的应用发展, 建 议应重视人工智能在医药健康领域发展的战略价值, 以解决国家人民健康重大需求为出发点, 明确数据基础设施和平台建设 为优先事项, 建立促进应用创新的交叉学科研究组织保障, 加强监管科学研究投入。

关键词: 医药健康; 人工智能; 国际比较

中图分类号: R-01 文献标识码: A

\section{Comparative Study on Global Development Strategies of Artificial Intelligence in Healthcare}

\section{Li Yingying ${ }^{1}$, Zhang Jiannan ${ }^{1}$, Gu Yanju ${ }^{1}$, Zhu Yelin ${ }^{1}$, He Qianfeng ${ }^{2}$, Li Lanjuan ${ }^{1,2}$}

(1. Institute of Medical-care Information Technology, Hangzhou 311100, China;

2. The First Affiliated Hospital of Zhejiang University, Hangzhou 310003, China)

\begin{abstract}
This study compares the artificial intelligence (AI) development strategies in healthcare in the US, Japan, UK, India, and China since 2015. Analysis shows that infrastructure investment and industrial layout are the core contents of these strategies. China's strategy is well laid out and ranks only second to the US in several aspects. However, China still faces several critical challenges, including lack of holistic planning and development paths, unclear focuses for development, lack of effective engagement of health data infrastructure with AI application, unbalanced structure of interdisciplinary talents, and insufficient regulation policies for AI application. To promote AI application in healthcare in China, we should clarify the strategic value of AI for healthcare advancement, focus on major health needs, prioritize the construction of data infrastructure and platforms, establish interdisciplinary research institutes to innovate AI application, and promote scientific researches on regulatory mechanisms.
\end{abstract}

Keywords: healthcare; artificial intelligence; international comparison

一、前言

人工智能 $(\mathrm{AI})$ 作为引领未来的战略性技术,
是新一轮产业变革的核心驱动力。世界主要发达国 家已把发展 $\mathrm{AI}$ 作为提升国家竞争力、维护国家安 全的重大战略。为抢占 $\mathrm{AI}$ 发展的国际领导地位,

收稿日期 : 2019-09-05; 修回日期 : 2019-09-12

通讯作者: 李兰娟, 浙江大学医学院附属第一医院教授, 中国工程院院士, 主要从事人工肝、传染病、数字医疗等的研究工作; E-mail: 1jli@zju.edu.cn

资助项目：中国工程院咨询项目 “人工智能在医药健康领域应用发展战略研究” (2019-ZD-6)

本刊网址：www.engineering.org.cn/ch/journal/sscae 
各国科技竞争愈演愈烈, 拥有科学前瞻的 $\mathrm{AI}$ 战略 布局将直接影响国家在国际竞争中的主动权, 对国 际政治经济格局等产生重大深远的影响。在医药健 康领域, AI 通过创新改造产业供给端, 优化存量、 补给增量, 能够解决当前突出的优质资源相对不足、 地区医疗服务水平差异大等问题, 推动关键药物、 医疗装备的研发智能化升级等。我国应尽早布局适 应国情的 “AI+ 医药健康” 国家战略计划, 对于 “健 康中国” “创新驱动发展” 的实施, 推进医药健康 产业成为国民经济新支柱的意义重大。

本文梳理了 2015 年至今世界主要国家 AI 发展 相关战略规划中涉及到医药健康领域的内容, 横向 比较分析了各国 “AI+ 医药健康” 战略布局特点, 推断 “ $\mathrm{AI}+$ 医药健康” 未来国际竞争趋势, 旨在为 我国 AI 在医药健康领域的战略发展规划提供参考 作用。

\section{二、各国在医药健康领域的人工智能战略 规划}

截至 2019 年 4 月, 至少已有 35 个国家出台了 $\mathrm{AI}$ 相关战略规划, 各国规划中均重视促进 AI 技术 在具体领域的应用和开发。其中美国、英国、日本、 印度和中国对于 AI 技术在医药健康领域的应用进 行了重点布局, 下面对这五个国家近 5 年来的相关 战略规划进行梳理, 重点分析其战略规划的布局重 点及演变过程。

\section{（一）美国在医药健康领域的人工智能战略规划}

早在 2016 年 10 月, 奥巴马政府出台的《为人 工智能的未来做好准备》中就提出了涉及管理、数 据、人才、研究、投资、政策等多方面的 23 条建 议措施, 其中指出联邦机构应该将用于 AI 的公开 训练数据及公开数据标准放在工作的首要位置 [1], 并在多条建议中强调了 $\mathrm{AI}$ 和数据科学的联系。同 年同月发布的《美国国家人工智能研究与发展战略 计划》中提到 $\mathrm{AI}$ 在医学领域的潜在价值在于: $\mathrm{AI}$ 可以支持生物信息系统, 从大规模基因组研究 (如 全基因组关联研究、测序研究) 中识别遗传风险, 并预测新药的安全性和有效性; AI 技术可以进行跨 多维数据评估, 用于公共卫生问题研究, 并为医疗 诊断和治疗提供决策支持; $\mathrm{AI}$ 是支持精准治疗所必
需的技术 $[2]$ 。

美国国立卫生研究院（NIH）具体负责整个美 国的医学研究管理, 在推动医药卫生领域 $\mathrm{AI}$ 发展 中, NIH 最先关注和开展了生物医学数据基础设施 的建设。2018 年 6 月, NIH 发布《NIH 数据科学战 略计划》提出机器学习、深度学习、AI 和虚拟现实 等技术创新可能在未来 10 年里为生物医学研究带 来革命性的变化, NIH 必须将其目前已有的数据科 学工作融入更大的数据生态系统中, 并充分利用已 有的和新兴的数据管理和技术成果、计算平台以及 各种创新工具 [3]。

2019 年以来, 美国联邦政府密集出台 AI 相关 政策, 加速推动 $\mathrm{AI}$ 应用发展。2 月, 《美国人工智 能计划》启动, 计划从五个重点方面入手来发展 $\mathrm{AI}$, 分别是：投资 $\mathrm{AI}$ 研发、释放 $\mathrm{AI}$ 资源、制定 $\mathrm{AI}$ 治理标准、增加 $\mathrm{AI}$ 从业者、国际参与, 以维持 美国在全球的 AI 优势; 6 月, 发布《国家人工智能 研究与发展战略计划: 2019 年更新版》, 在更新原 七项战略的基础上新增第八项战略：通过扩大政府 和社会资本合作模式, 加速 AI 发展 [4]。在第二项 战略一一开发有效的人机协作方法中, 对于医疗领 域, NIH 正在重点推进医学领域自然语言处理方面 的研究, 该研究的基础是美国国家医学图书馆保存 的所有 MEDLINE 引文中提取出的 9630 万个事实 构成的数据库。除此之外, 其他战略要点中也强调 了医疗健康领域公共数据集及 $\mathrm{AI}$ 应用于医疗这类 公共领域时的标准化及评估体系建设。

\section{（二）英国在医药健康领域的人工智能战略规划}

2017 年 10 月，英国政府发布《产业战略：创 建适应未来的英国》, 确立 $\mathrm{AI}$ 和数据经济发展的四 个优先领域: 将英国建设为全球 $\mathrm{AI}$ 与数据创新中 心; 支持各行业利用 $\mathrm{AI}$ 和数据分析技术; 在数据 和 $\mathrm{AI}$ 的安全等方面保持世界领先; 培养公民工作 技能 [5]。2018 年 4 月，英国发布政策文件《产业 战略: 人工智能领域的协议》, 旨在推动英国成为 全球 $\mathrm{AI}$ 领导者, 该战略从目标、人才、基础设施、 商业环境、区域发展五个重点方面进行布局：英国 政府拟投入 4.06 亿英镑用于科学、技术、工程、数 学教育投资; 拟投资超过 10 亿英镑用于改善数字 基础设施的建设, 着力解决数据开放, 实现数据可 用性的提升; 英国政府还将建立新的 $\mathrm{AI}$ 理事会、 
$\mathrm{AI}$ 办公室以及数据伦理与创新中心 $(\mathrm{CDEI})$ 。其中, $\mathrm{CDEI}$ 是英国政府 $\mathrm{AI}$ 计划的重要组成部分, 英国希 望以此引领全球 $\mathrm{AI}$ 道德研究, 促使英国成为全球 $\mathrm{AI}$ 的领导者。

在医疗健康领域, 该政策提到 2.1 亿英镑的产 业战略挑战基金（ISCF）将用于支持数据应用于早 期诊断和精准医学 (包括使用 AI 分析数字病理医学 图像) 的研究, 并在后续政策中持续推进。2018 年 11 月, 英国政府宣布拨款 5000 万英镑用于更深入 地开发 $\mathrm{AI}$ 在医疗细分领域的应用, 来提升癌症等 多种疾病的早期诊断能力和病患护理效率, 并专门 成立了 5 个 AI 医疗技术中心来联合研究机构和企 业共同开发更智能的医学成像分析应用, 为患者做 出更好的临床决策。此外, 2019 年 2 月, 英国国 家卫生服务系统（NHS）宣布将组建新的联合单位 NHSX, 用于加速 NHS 的数字化转型。2019 年 8 月, $\mathrm{NHS}$ 投资 2.5 亿英镑建立国家 $\mathrm{AI}$ 实验室, 推动 $\mathrm{AI}$ 技术在英国医疗领域的应用。

\section{（三）日本在医药健康领域的人工智能战略规划}

日本是全球第二个制定国家层面 $\mathrm{AI}$ 战略的国 家, 在此之前日本政府科技重点已围绕 “机器人驱 动的新工业革命” 展开。2016 年 1 月, 日本政府提 出要在世界上率先建立高度融合网络空间和物理空 间的 “超智能社会” (Society 5.0), 并在世界范围内 产生重大影响 [6]。2017 年 3 月, 日本发布《人工
智能技术战略》, 指出 $\mathrm{AI}$ 技术与其他相关技术的融 合为解决各种社会问题提供了可能性。战略提出 $\mathrm{AI}$ 技术应作为一项服务, 即 “AI as a service (AIaaS)”, 通过与不同类型的数据结合, 把 $\mathrm{AI}$ 技术应用和拓 展到各个领域中, 因此日本提出的是 $\mathrm{AI}$ 与其他相 关技术融合的产业化路线图, 并且在各个阶段中均 强调了数据的重要作用。健康、医疗、福利作为重 点发展的三大领域之一, 战略规划中给出了该领域 的详细产业化发展路线图, 日本也是本研究涉及的 国家中唯一给出明确的医药健康领域发展路径的国 家（见图 1) [7]。对于发展 AI 在医药健康领域的 应用, 日本的发展目标非常明确, 即建立一个享有 健康生活和长寿的社会, 旨在推进疾病的预防, 使 日本成为健康和长寿行业的领导者。其中, 数据基 础设施建设是最先启动的环节, 医疗、护理机器人 的逐步智能化提升以及围绕个人更加便捷、智能的 健康管理与医疗服务是其发展重心。在 2017 年财 年预算中, 厚生劳动省分别拨付 4.7 亿日元、 1.8 亿 日元用于临床 $\mathrm{AI}$ 数据系统实证研究和利用 $\mathrm{AI}$ 支持 新药研发活动 [8]。

\section{（四）印度在医药健康领域的人工智能战略规划}

为应对中国 $\mathrm{AI}$ 的快速发展, 推动印度在 $\mathrm{AI}$ 领 域的发展, 印度总理纳伦德拉 - 莫迪要求印度智库 Niti Aayog 研究利用 AI 技术解决该国社会经济等 问题。2018 年 6 月, Niti Aayog 制定发布了《国家
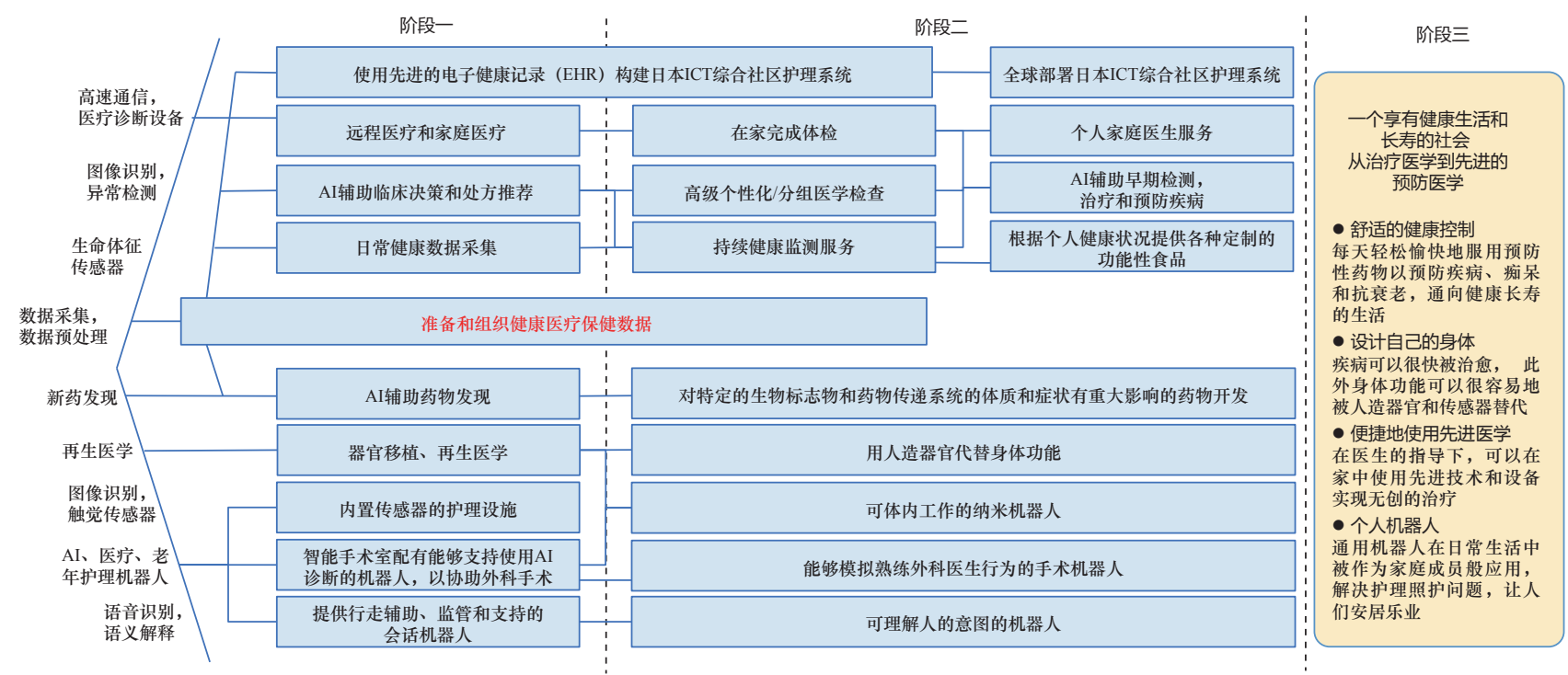

图 1 日本人工智能与其他相关技术融合的产业化路线图（卫生、医疗、福利） 
人工智能战略 (讨论稿)》, 在该报告中提出了 “ $\mathrm{AI}$ for all” 战略, 旨在利用 AI 促进经济增长、社会发 展和包容性增长, 使印度成为新兴经济体和发展 中经济体的 “创新实验地”, 确保印度在全球经济 中发挥更大的作用, 以获得其在转型时代中应有的 地位 [9]。战略指出, 卓越的研究能力是新兴技术 领导的基础, 释放技术带来的增长潜力需要基础 研究和应用研究, 因此建议建立 “AI 卓越研究中 心” (CORE) 和 “国际 AI 转化中心”( ICTAI) 两 类中心来推动印度 $\mathrm{AI}$ 发展。其中, CORE 专注于 基础研究并作为 ICTAI 的技术供应商, 而 ICTAI 则 将专注于技术的市场转化和社会重要领域的 AI 应 用研发。2019 年 5 月, Niti Aayog一项递交至财务 部的提案计划提出未来 3 年内投入 7500 亿卢比用 于建立分析和知识同化平台 (AIRAWAT), 通过通 用云平台的建立来支持目前印度国内的各项研究和 开发。同时, 战略还提出, 政府在发展 $\mathrm{AI}$ 中要扮 演积极的推动者角色, 要将 $\mathrm{AI}$ 应用于整个价值链, 考虑借鉴市场化机制构建全国的 AI 市场（NAIM）, 专注于数据采集汇聚、数据标注和模型部署, 促进 各个利益相关方的参与, 以简化协作, 减少收集和 注释数据的时间和成本, 并实现多个解决方案的统 一部署, 以实现规模和网络效果。

在该战略中, 医疗保健是其优先考虑发展的领 域, 核心目标是提高高质量医疗保健服务的可及性 和可负担性。Niti Aayog 正在讨论启动建立一个具 有标注和分类功能的国家级病理图像数据库和癌症 影像生物数据库, 希望利用 $\mathrm{AI}$ 技术来降低成本, 提供临床决策支持以改善癌症诊疗效果。

\section{（五）中国在医药健康领域的人工智能战略规划}

2015 年 7 月, 我国发布《国务院关于积极推进 “互联网 + ” 行动的指导意见》, 已将 $\mathrm{AI}$ 作为重点 布局领域之一，提出要建设支撑超大规模深度学习 的新型计算集群 (海量训练资源库), 加强 $\mathrm{AI}$ 基础 资源和公共服务等创新平台建设, 进一步推进关键 技术的研发和产业化。2017 年 7 月，国务院发布《新 一代人工智能发展规划》, 该规划是中国 $\mathrm{AI}$ 专项长 期发展规划, 在重点任务建设安全便捷的智能社会 里提出要发展便捷高效的智能服务, 其中包括智能 医疗、智能健康和养老等, 并提出在健康保障等重 大项目中加强 AI 技术应用示范 [10]。2017 年 12 月,
工业和信息化部发布了《促进新一代人工智能产业 发展三年行动计划（2018-2020 年)》, 提出要在 若干重点领域形成国际竞争优势, 医疗领域的重点 为扩大医疗影像辅助诊断等系统的临床应用 [11]。 2019 年 6 月, 科学技术部发布《新一代人工智能治 理原则》, 提出要发展负责任的 $\mathrm{AI}$, 符合人类的价 值观和伦理道德, 避免误用, 禁止滥用、恶用 [12]。 2019 年 8 月, 科学技术部发布《国家新一代人工智 能开放创新平台建设工作指引》的通知, 围绕《新 一代人工智能发展规划》重点任务中涉及的具有重 大应用需求的细分领域组织建设, 原则上每个具体 细分领域建设一家国家新一代 AI 开放创新平台 [13]。

具体到医药健康领域, 2017 年 1 月, 国家卫 生健康委员会发布《“十三五”全国人口健康信息 化发展规划》, 提出要发挥 AI、医用机器人等先 进技术和装备产品在人口健康信息化和健康医疗 大数据应用发展中的引领作用。2018 年 5 月, 国 务院办公厅发布《关于促进 “互联网 + 医疗健康” 发展的意见》, 提出要推进 “互联网 + ” $\mathrm{AI}$ 应用服 务; 研发基于 $\mathrm{AI}$ 的临床诊疗决策支持系统; 加强 临床、科研数据整合共享和应用; 支持研发医疗 健康相关的 AI 技术、医用机器人、大型医疗设备、 应急救援医疗设备、生物材料三维打印成型技术 和可穿戴设备等 [14]。

\section{三、各国在医药健康领域的人工智能战略规 划比较分析}

五个国家的医药健康领域的国家 $\mathrm{AI}$ 战略发展 规划比较显示 (见表 1), 各国均从本国实际国情和 产业优势出发制定相应的 AI 发展策略, 规划要点 集中于：数据和基础设施平台建设、创新研究组织 搭建、重点领域布局、人才队伍建设及配套治理体 系完善。同时，各国的 $\mathrm{AI}$ 发展政策与其原有的科 技发展战略也保持密切的联系，这样的结合既有利 于突出 $\mathrm{AI}$ 在不同领域的应用重点, 也有利于充分 利用各国在特定领域的已有优势，短时间内尽可能 地释放 $\mathrm{AI}$ 在该领域的生产潜力 [15]。

\section{(一) “AI+ 医药健康” 规划特点比较}

在本文研究的五个国家中, 美国具有非常明确 的 $\mathrm{AI}$ 战略发展体系, 主要以《国家人工智能研究 
表 1 医药健康领域的国家人工智能战略发展规划比较

\begin{tabular}{|c|c|c|c|}
\hline 国家 & 国情需求 & 重点任务 & 基础设施 \\
\hline 美国 & $\begin{array}{l}\text { 美国医疗系统总体上 “重治疗, 轻预 } \\
\text { 防”, 医疗卫生费用支出处于全球领先, } \\
\text { 而平均期望寿命低于经济发展与合作 } \\
\text { 组织成员国家, 且近年来多次出现下降 }\end{array}$ & $\begin{array}{l}\text { 医疗诊断、手术机器人、AI } \\
\text { 医疗可穿戴设备 }\end{array}$ & $\begin{array}{l}\text { 数据: 重点发展数据基础建设, 构建 } \\
\text { 生物医学数据科学现代化生态系统; } \\
\text { 开发共享公共数据集, 增加对数据集 } \\
\text { 的访问 }\end{array}$ \\
\hline 英国 & $\begin{array}{l}\text { 医疗服务供不应求, 医疗费用不断上 } \\
\text { 升, 医务人员积极性不高, 病人排队 } \\
\text { 住院时间太长, 医疗资源向私立医院 } \\
\text { 转, 投入了很高的经费但是没有达 } \\
\text { 到预期的结果, 国民对医疗保障的不 } \\
\text { 满加剧 }\end{array}$ & $\begin{array}{l}\text { 提升癌症等多种疾病早期诊 } \\
\text { 断和病患护理效率、影像诊断 }\end{array}$ & $\begin{array}{l}\text { 数据: 建设强大的数字和电信基础设 } \\
\text { 施, 启动数据伦理与创新中心; 改进 } \\
\text { 现有数据基础设施, 发布更高质量的 } \\
\text { 公共数据。 } \\
\text { 平台: 医学 } \mathrm{AI} \text { 技术中心; 国家 } \mathrm{AI} \text { 实 } \\
\text { 验至 }\end{array}$ \\
\hline 日本 & $\begin{array}{l}\text { 少子化、高龄化、人口减少; 是世界上 } \\
\text { 老龄化最迅速的国家, 到 } 2030 \text { 年, 超 } \\
\text { 过 } 40 \% \text { 的日本人口将是老年人, 社会 } \\
\text { 保障开支较大, 劳动力不断减少。此外, } \\
\text { 日本地震频发, 受自然灾害影响的救 } \\
\text { 助工作量较大 }\end{array}$ & $\begin{array}{l}\text { 痴呆等疾病早期发现、医疗 } \\
\text { 诊断设备、约物发现、染色 } \\
\text { 体笚查、辅助诊治、手术机 } \\
\text { 器人、护理机器人 }\end{array}$ & $\begin{array}{l}\text { 数据: 准备 AI 性能评估数据, 如标 } \\
\text { 准图片数据集; 加强专门负责数据 } \\
\text { 维护的机构系统 (NICT, JST、 RIK- } \\
\text { EN); 由 由ICT、RIKEN、AIST 今 } \\
\text { 中心负责数据维护和与卫生部门数据 } \\
\text { 协调 } \\
\text { 平台: 开发模拟现实的仿真环境; 提 } \\
\text { 供 AI 云与基于云的开放工具。 }\end{array}$ \\
\hline 印度 & $\begin{array}{l}\text { 国内卫生保健可及性差距大, 医疗服 } \\
\text { 务的质量不平衡, 印度农村和城市之 } \\
\text { 间差距明显。缺乏合格的卫生人员和 } \\
\text { 基础设施。个人医疗支出占医疗支 } \\
\text { 出的 } 70 \% \text {, 是自付比最高的国家之一 }\end{array}$ & $\begin{array}{l}\text { 个性化治疗、早期识别潜在 } \\
\text { 流行病和成像诊断、预防技术 }\end{array}$ & $\begin{array}{l}\text { 数据: 启动病理图像数据库, 提供高 } \\
\text { 质量已标注的病理数据集; 讨论建立 } \\
\text { 癌症成像生物库, 用以研究癌症表型 } \\
\text { 的成像相关性; } \\
\text { 平台: 建立 AIRAWAT 云平台 }\end{array}$ \\
\hline 中国 & $\begin{array}{l}\text { 老龄化加剧, 到 } 2020 \text { 年 } 60 \text { 岁及以上 } \\
\text { 老龄人口将达到 } 2.55 \text { 亿, 占人口的 } \\
\text { 约 } 17.8 \% \text { 。慢性非传染性疾病成为主要 } \\
\text { 疾病负担; 缺乏优质的卫生人员, 缺 } \\
\text { 之自主可控的高端医疗装备 }\end{array}$ & $\begin{array}{l}\text { 机器智能辅助个性化诊断、 } \\
\text { 精准治疗辅助决策支持系统、 } \\
\text { 智能医疗健康设备、辅助康 } \\
\text { 复和照看、手术机器人、智 } \\
\text { 能护理机器人 }\end{array}$ & $\begin{array}{l}\text { 数据: 互联互通的人口健康信息平台; } \\
\text { 超大规模深度学习的新型计算集群, } \\
\text { 海量训练资源库; } \\
\text { 平台: 加强 } \mathrm{AI} \text { 基础资源和公共服务等 } \\
\text { 创新平台建设; 创建细分领域 } \mathrm{AI} \text { 创新 } \\
\text { 平台 }\end{array}$ \\
\hline
\end{tabular}

和发展战略计划》搭建了美国推动 $\mathrm{AI}$ 发展的战略 实施框架，并由 $\mathrm{NIH}$ 负责医药领域的 AI 研发投入 与管理。英国 $\mathrm{AI}$ 战略规划从属于英国产业战略之 下, 且特别强调将 $\mathrm{AI}$ 的发展与数字经济战略相结 合, 在《产业战略：建设适应未来的英国》和《产 业战略: 人工智能领域协议》等多份政策文件中, 数字经济发展与 $\mathrm{AI}$ 均关联出现, 医药健康领域的 发展也延续了这一特征, NHS 将组建 NHSX 推动 健康医疗数据化进程。日本依托其在智能机器人领 域的全球领先地位, 致力于通过机器人驱动革命, 医药健康领域 $\mathrm{AI}$ 发展战略中也以此为重心。印度 期望利用 AI 获取更高的全球影响力, 目前主要由 Niti Aayog 负责推进研究基础设施建设以及医药健 康领域 $\mathrm{AI}$ 应用试点。

我国同样拥有整体性的战略部署，通过《新一 代人工智能发展战略规划》统筹指导新一代 $\mathrm{AI}$ 的 发展, 规划中涉及的产业面非常完整, 但建设重点 不够突出。

\section{（二）“AI+ 医药健康” 重点任务比较}

作为关乎民生的核心领域, 各国在医药健康领 域确定重点发展任务的策略中, 主要从本国国情需 求出发, 并考虑已有优势的健康医疗产业升级为主。 目前全球面临着普遍的卫生问题: 老龄化趋势严 重、慢性病增加、全球性传染病风险、医疗保障体 系负担加剧等, 因此各国在医药健康领域的 $\mathrm{AI}$ 应 用布局上非常相似, 重点内容集中在疾病诊断设备、 疾病早期识别、药物研发、癌症治疗、手术机器人、 辅助机器人等。但依据国情不同优先级别有所差异, 如日本面临着严重的少子化和老龄化，导致医疗、 福利等社会保障负担严峻, 护理人员面临严重短缺, 同时日本地震等自然灾害繁多，因此在其战略发展 中明确提出 $\mathrm{AI}$ 在应对老龄化问题的战略地位，优 先发展利用 $\mathrm{AI}$ 解决医疗健康劳动力的问题, 研发 领域中还提及搜救机器人等。印度作为肿瘤治疗仿 制药生产大国, 重视 AI 在肿瘤治疗领域的应用发 展，如能依其战略良性发展，不排除在未来印度对 
全球创新制药产业格局形成冲击的可能。

中国医疗资源分布不平衡问题突出, 在重点任 务中强调了 “互联网 + ” 与 $\mathrm{AI}$ 战略衔接以延伸服 务空间, 实现将中国 “互联网 +” 产业优势转化到 $\mathrm{AI}$ 应用发展中。

\section{（三）“AI+ 医药健康” 基础设施投入比较}

在各国战略规划中，基础设施建设投入，尤其 是数据层面的基础设施建设投入, 往往是战略实施 路径中的第一阶段内容。健康医疗数据基础条件较 好的国家如美国、英国均强调在现有数据基础上建 立更加共享开放的公共数据集, 而原有数据基础相 对不足的印度则采取建立垂直领域数据库如肿瘤影 像数据库、癌症生物数据库等。研究中心是研发和 提供持续的创新动力的基础保障, 英国重视各类研 究中心、联合研究机构的组建以保持 $\mathrm{AI}$ 研发能力 的领先, 印度强调基础研究与应用研究, 强调建立 两类研究中心并明确两者的关系。在平台建设层面, 各国各具特色, 日本提出要建立开发模拟现实的仿 真环境; 印度建立通用云平台实现组织联络与资源 共享; 我国提出了搭建新一代 $\mathrm{AI}$ 开放创新平台推 动技术创新与成果转化应用。

\section{（四）我国 “A I+ 医药健康” 战略布局关键差距}

总结各国战略规划中的领先布局并分析我国的 关键差距（见表 2) 显示: 在医药健康领域的体系 框架上, 与领先国家体系性的战略布局相比, 我国 缺乏清晰的 $\mathrm{AI}$ 在医药健康领域的发展路径, 公共 投入与企业投入以及应用的产研重点不明确, 并且 战略的联动性不足, 在推动 $\mathrm{AI}$ 与实体经济融合过 程中, 更多停留于推动 $\mathrm{AI}$ 在各个领域的具体应用, 而尚未明确指出其与供给侧结构性改革这一经济体
系改革的核心战略关系 [15], AI 对于推动健康医疗 供给侧变革的重要战略价值还未凸显。在基础设施 建设上, 美国的医药健康 $\mathrm{AI}$ 战略与数据科学发展 形成有效联动, 使其完善的健康医疗数据基础设施 优势延续到了 $\mathrm{AI}$ 战略布局中, 英国对于基础建设 的财政投入明确, 相对而言, 我国虽然也强调了数 据基础设施建设, 但现有的人口健康信息平台建设 分散, 未与医学科学发展、 $\mathrm{AI}$ 应用等形成有效的衔 接和联动。在人才梯队建设上, 尚未对医药与 $\mathrm{AI}$ 领域复合人才结构失衡的问题予以重视。此外, 美 国完善的制度管理体系和监管科学研究, 能够较快 地对医药健康 AI 应用做出调整和适应, 进而对产 业有序发展形成有效支撑, 我国虽然拥有自上而下 的迅速调整能力, 但在监管科学建设上还存在明显 不足。最后, 虽然我国同样重视在 AI 发展过程中 的重点伦理与安全问题, 但相应的标准规范和法律 法规基础与英、美相比差距明显, 在这方面的标准 制定缺乏相当的国际话语权。

\section{四、我国 “AI+医药健康” 战略规划重点内 容建议}

\section{（一）重视 AI 在医药健康领域发展的战略价值}

“健康中国 2030” 对我国医药健康领域发展提 出了更高的要求, 我国要在 2030 年基本实现健康 公平, 主要健康指标进入高收入国家行列。人民健 康是民族昌盛和国家富强的重要标志, 在人口老龄 化、环境变化、生活行为方式转变趋势下, 我国各 年龄段医疗健康需求急剧增加, 患病人群年轻化与 精神疾病高发威胁着中国劳动人口健康, 慢性非传 染性疾病成为居民主要死因和疾病负担。而我国现 有医疗健康服务供给体系结构失衡、总量不足、增

表 2 中国医药健康领域的国家人工智能战略发展规划的关键差距

\begin{tabular}{llll}
\hline 规划内容 & 领先国家 & \multicolumn{1}{c}{ 领先布局 } & \multicolumn{1}{c}{ 中国关键差距点 } \\
\hline 体系框架 & 日本、美国 & 整体医药 $\mathrm{AI}$ 体系化发展框架与路径 & 缺乏医药 $\mathrm{AI}$ 总体规划统筹引导 \\
技术发展 & 美国、中国 & 重视基础研究投入, 建立开放创新平台 & \\
基础设施 & 美国、英国 & $\mathrm{AI}$ 健康医疗数据集; 大型算力基础设施 & $\begin{array}{l}\text { 缺乏医药健康领域数据基础建设与 } \mathrm{AI} \text { 数据基础 } \\
\\
\end{array}$ \\
& & 设施建设的衔接, 领域算力投入关注不足 \\
人才保障 & 美国 & $\mathrm{AI}$ 人才梯度建设; 首席科学家团队建设 & 未重视医药与 $\mathrm{AI}$ 领域复合人才的结构失衡 \\
审批监管 & 美国 & 监管科学研究 & 无适用 $\mathrm{AI}$ 的审批监管机制 \\
隐私安全 & 英国 & 标准规范; 法律法规 & 医疗健康数据资产缺乏立法保障, 伦理安全制度 \\
& & & 基础薄弱 \\
\hline
\end{tabular}


速有限, 关键药物、医疗器械研发创新短板明显, 亟需理念创新、技术创新、系统创新。建议应设立 医疗健康 AI 应用发展专项计划, 明确发展路径, 吸收各国先进经验, 推动 $\mathrm{AI}$ 在医药健康领域的应 用发展, 并重视与已有产业战略规划、整体经济 科技战略的协调。

\section{（二）以解决国家人民健康的重大需求为出发点}

纵观主要国家重点领域, 核心均是围绕各国医 药健康领域的国情出发。针对中国卫生领域的国情 背景, 建议应以健康促进、人口老龄化应对为核心, 围绕医药健康领域难题, 重点推进 AI 在临床诊断、 治疗、创新药物研发、精准健康管理、合理医保控 费等领域的应用。同时, 医疗装备是现代医学不可 缺少的重要工具, 而中国医疗装备长期依赖进口, $\mathrm{AI}$ 技术给医疗装备制造带来了产业变革的历史机 遇, 把握住医疗装备智能化升级的关键时期, 我国 有可能实现 “换道超车”, 因此建议加强以医疗器 械等高端装备国产替代升级为主线的智能化产业发 展的扶植力度。

\section{（三）明确医药健康数据基础设施和平台建设为优 先事项}

医药健康数据是 $\mathrm{AI}$ 应用发展的基础必要设施, 全球范围内的主要国家均将其视为战略发展优先事 项, 中国拥有全球第一的健康医疗数据资源, 但在 资源可用性上与其他优势国家相比存在明显差距, 激活数据驱动的医药 $\mathrm{AI}$ 应用价值需要将现有的医 药健康数据规划和 $\mathrm{AI}$ 发展战略形成有效的衔接, 优先发展适用于 $\mathrm{AI}$ 发展的医药健康数据基础设施 建设, 加速资源价值转换, 建议考虑一把手推进国 家医药健康大数据共享平台建设, 重点解决数据质 量和数据共享两大难题, 并重视数据治理过程, 重 点加强信息标准化建设。同时 “ $\mathrm{AI}+$ 医药健康” 是 目前市场活跃度最高的领域之一, 建议在创新共享 平台建设中优先考虑医药健康细分领域的建设, 加 快引导产业有序发展。

\section{（四）建立促进应用创新的 “AI+ 医药健康” 交叉学 科研究组织}

基于大数据和 $\mathrm{AI}$ 的新科学研究范式正在形成, 需要更紧密的学科交叉融合。建立交叉研究中心成
为多个国家的战略选择。 $\mathrm{AI}$ 在医药健康领域的应用 关键是要医药健康领域专家的参与, 为推动该领域 的持续创新需要有稳定的组织保障, 建议可依托国 家医学研究中心等医学领域研究机构, 成立一系列 重点疾病领域的医疗 AI 交叉研究中心, 建立有效 的 “产学研用” 转化机制, 加速交叉人才培养, 促 进智能化诊断、治疗和健康管理应用的研发, 提高 $\mathrm{AI}$ 技术下新药研发的成功率和转化率。

\section{（五）加强 AI 在医药健康领域应用的监管科学研究 投入}

战略规划核心是在促进 $\mathrm{AI}$ 发展的同时控制其 所引发的治理风险。医疗健康服务和应用的质量与 安全直接关系到人民群众的生命健康, “AI+医药 健康” 应用同样也需要受到行业的严格监管。目前 监管体制的滞后一定程度上限制了产业发展。应加 强监管科学研究投入, 为建立完善覆盖创新服务与 应用产品全生命周期的包容、审慎、有效的监管机 制提供科学依据。

致谢

感谢中国工程院咨询项目 “人工智能在医药健康领域应用发展 战略研究” 项目组各位院士和专家对本次研究的指导。

\section{参考文献}

[1] 美国国家科技委员会的机器学习与人工智能分委会. 美国白宫 报告: 为人工智能的未来做好准备 [J]. 信息安全与通信保密, 2016 (12): 9-14.

Executive Office of the President National Science and Technology Council Committee on Technology. White House report: Preparing for the future of artificial intelligence [J]. Information Security and Communications Privacy, 2016 (12): 9-14.

[2] National Science and Technology Council. The national artificial intelligence research and development strategic plan [R]. Washington DC: National Science and Technology Council, 2016.

[3] National Institutes of Health. NIH strategic plan for data science [R]. Bethesda: National Institutes of Health, 2018.

[4] Select Committee on Artificial Intelligence of the National Science \& Technology Council. The national artificial intelligence research and development strategic plan: 2019 update [R]. Washington DC: Select Committee on Artificial Intelligence of the National Science \& Technology Council, 2019.

[5] Energy Department for Business, Industrial Strategy. Industrial strategy: Building a Britain fit for the future [R]. London: Energy Department for Business, Industrial Strategy, 2017.

[6] 朱启超, 王姝. 日本“超智能社会”建设构想: 内涵、挑战与影响 [J]. 日本学刊, 2018 (2): 60-86.

Zhu Q C, Wang S. Japan's "Society 5.0": The strategic vision, 
challenges and influence [J]. Japanese Studies, 2018 (2): 60-86.

[7] Strategic Council for AI Technology. AI technology strategy [R]. Tokyo: Strategic Council for AI Technology, 2017.

[8] 中国信息通信研究院, 中国人工智能产业发展联盟. 全球人工 智能战略与政策观察(2019) [R]. 北京: 中国信息通信研究院, 中 国人工智能产业发展联盟, 2019.

China Academy of Information and Communication Technology, China Artificial Intelligence Industry Innovation Alliance. The observing of artificial intelligence strategy and policy (2019) [R]. Beijing: China Academy of Information and Communication Technology, China Artificial Intelligence Industry Innovation Alliance, 2019.

[9] NITI Aayog. National strategy for AI discussion paper [R]. New Delhi: NITI Aayog, 2018.

[10] 中华人民共和国国务院. 新一代人工智能发展规划 [R]. 北京: 中华人民共和国国务院, 2017.

State Council of the PRC. The plan for development of the new generation of artificial intelligence [R]. Beijing: State Council of the PRC, 2017.

[11］中华人民共和国工业和信息化部. 促进新一代人工智能产业发 展三年行动计划(2018-2020年) [R]. 北京: 中华人民共和国工 业和信息化部, 2017.

Ministry of Industry and Information Technology of the PRC. A three-year action plan to develop AI (2018-2020) [R]. Beijing: Ministry of Industry and Information Technology of the PRC,
2017.

[12] 国家新一代人工智能治理专业委员会. 新一代人工智能治理原 则——发展负责任的人工智能 [R]. 北京: 国家新一代人工智能 治理专业委员会, 2019 .

China's National Professional Committee on Next Generation AI Governance. Principles of the next generation AI governanceDevelop responsible AI [R]. Beijing: China's National Professional Committee on Next Generation AI Governance, 2019.

[13] 中华人民共和国科学技术部. 国家新一代人工智能开放创新平 台建设工作指引 [R]. 北京: 中华人民共和国科学技术部, 2019 .

Ministry of Science and Technology of the PRC. Guidelines for the construction of the new generation of $\mathrm{AI}$ innovation platforms [R]. Beijing: Ministry of Science and Technology of the PRC, 2019 .

[14] 中华人民共和国国务院办公厅. 国务院办公厅关于促进“互联 网+医疗健康”发展的意见 [R]. 北京: 中华人民共和国国务院办 公厅, 2018.

General Office of the State Council of the PRC. Opinions on promoting the development of "Internet Plus Healthcare" [R]. Beijing: General Office of the State Council of the PRC, 2018.

[15] 贾开, 郭雨晖, 雷鸿竹, 等. 人工智能公共政策的国际比较研究: 历史、特征与启示 [J]. 电子政务, 2018 (9): 78-86.

Jia K, Guo Y H, Lei H Z, et al. Comparative study on international public policy of artificial intelligence: History, characteristics and enlightenment [J]. E-Government, 2018 (9): 78-86. 